\title{
MTS image analyzer: a software tool to identify mesial temporal sclerosis in MRI images
}

Castillo, D., Macas, J., Samaniego, R., Jiménez, Y., Rodríguez-Álvarez, M. J., et al.

D. Castillo, J. Macas, R. Samaniego, Y. Jiménez, M. J. Rodríguez-Álvarez, Vasudevan Lakshminarayanan, "MTS image analyzer: a software tool to identify mesial temporal sclerosis in MRI images," Proc. SPIE 11842, Applications of Digital Image Processing XLIV, 1184215 (1 August 2021); doi: 10.1117/12.2594199

Event: SPIE Optical Engineering + Applications, 2021, San Diego, California, United States 


\title{
MTS Image Analyzer: A Software Tool To Identify Mesial Temporal Sclerosis In MRI Images
}

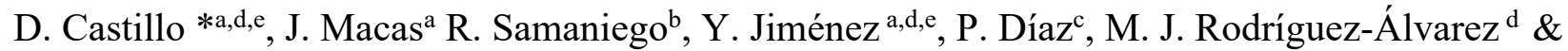 \\ Vasudevan Lakshminarayanan ${ }^{\mathrm{e}, \mathrm{f}}$ \\ ${ }^{a}$ Chemistry Department, Universidad Técnica Particular de Loja, Ap. 11-01-608, Loja- Ecuador; \\ ${ }^{b}$ Dept. of Radiology, Hospital UTPL, Loja-Ecuador; 'Health Sciences Department, Universidad \\ Técnica Particular de Loja, Ecuador; 'Instituto de Instrumentación para Imagen Molecular (i3M) \\ Universitat Politècnica de València - Consejo Superior de Investigaciones Científicas (CSIC), \\ Valencia, Spain; ${ }^{\mathrm{e} T h e o r e t i c a l ~ a n d ~ E x p e r i m e n t a l ~ E p i s t e m o l o g y ~ L a b, ~ S c h o o l ~ o f ~ O p t o m e t r y ~ a n d ~ V i s i o n ~}$

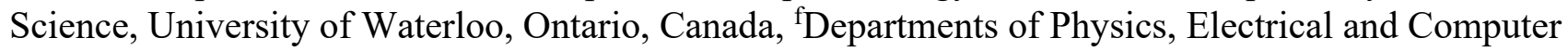 \\ Engineering and Systems Design Engineering, University of Waterloo, Ontario, Canada
}

\begin{abstract}
Epilepsy is a chronic neurological disorder that causes unprovoked and recurrent seizures which according to WHO affects approximately 50 million people worldwide. Functional magnetic resonance images (MRI) help to identify certain affected areas of the brain, namely, the gliosis and hippocampal volume loss. These losses cause complex epilepsy, and is known as hippocampal sclerosis or Mesial Temporal Sclerosis (MTS). This work presents the development of a Computer Aided Diagnosis CAD system software package) that can be used to identify the characteristics and patterns of MTS from brain magnetic resonance images. The image processing techniques involve texture analysis, statistical features, evaluation of the 3D Region of interest (ROI), and threshold analysis. The software allows the automatic evaluation of the degeneration of hippocampal structures, hippocampal volume and signal intensity. We will describe and demonstrate the software (which can currently be accessed on GitHub). It is expected that this tool will be useful in new neurology/radiology specialists and can serve as a secondary diagnosis. However, it is necessary to validate the software system qualitatively and quantitatively in order to get more effectiveness and efficiency in a real-world clinical application.
\end{abstract}

Keywords: brain MRI, Mesial temporal sclerosis, hippocampal volume, epilepsy, texture features, 3D ROI, texture analysis

\section{INTRODUCTION}

According to World Health Organization ${ }^{1}$ (WHO), epilepsy is a chronic neurological disorder that causes unprovoked and recurrent seizures which affect approximately more than 50 million people in the world and $80 \%$ of that live in low and middle income countries. It is estimated that $70 \%$ of people living with epilepsy could live seizure free if properly diagnosed and treated ${ }^{1}$. Epilepsy also is associated with hippocampal sclerosis (HS) ${ }^{2}$ which is the most common cause of mesial temporal lobe epilepsy ${ }^{3}$ as well as other epilepsy syndromes. HS is also referred Mesial temporal sclerosis (MTS) and is found in drug resistant, chronic temporal lobe epilepsies ${ }^{4,5}$. This condition is described by loss and chronic fibrillary gliosis in the pyramidal cell layer of the hippocampus ${ }^{2}$.

MTS can be identified by functional Magnetic Resonance Image (MRI), which allows for the evaluation and assessment of hippocampal deformation ${ }^{6}$. The coronal volume and coronal high resolution of T1-weighted with inversion recovery (IR), and T2-weighted, and the fluid-attenuated inversion recovery (FLAIR) modalities of MRI are considered to be the crucial images to best diagnose $\mathrm{MTS}^{7-9}$. More specifically, the FLAIR modality allows assessment signal intensity. ${ }^{4,10,11}$

There is great variability in visual inspection of MRI images of MTS in MRI according to Silva et al. ${ }^{11}$ The variability

*dpcastillo@utpl.edu.ec; phone +593 07370 1444; ext.3204; www.utpl.edu.ec

Applications of Digital Image Processing XLIV, edited by Andrew G. Tescher, Touradj Ebrahimi, Proc. of SPIE Vol. 11842, 1184215 - @ 2021 SPIE · CCC code: 0277-786X/21/\$21 · doi: 10.1117/12.2594199 
ranges between $62 \%$ and $85 \%^{7,11,12}$. For this reason an experienced radiologist is needed to accurately discern the changes of the hippocampal structures and abnormal signals. ${ }^{7,13,14}$

In this context, artificial intelligence, namely Machine Learning/Deep Learning could help analyze and diagnose the MR brain images ${ }^{15-19}$ as well as other parts of the human body. There is a need to detect and classify MTS images and the development of systems that could assess, detect and identify the changes of the hippocampal structures like the volume and intensity of signals..$^{9,11,13,14,20}$ There is some literature ${ }^{10,19,21,22}$ dealing with the automatic detection of the hippocampus. These systems employ the fact that there is hippocampal volume loss which is a principal feature of MTS $^{14}$. Jafari et al. ${ }^{23}$ detected the hippocampus through the analysis of signal and texture features in FLAIR images using a machine learning framework. Cantor et al. ${ }^{24}$ have proposed the detection of temporal lobe epilepsy in multiparametric quantitative MRI T1 and T2 maps, using Support Vector Machine (SVM), feature selection and, Principal Component Analysis (PCA) method. They reported an accuracy of $88.9 \%$. In addition, using SVM and a large cohort of epilepsy patients with and without mesial temporal sclerosis (MTS), Rudie et al..$^{25}$ achieved an automatic classification of MTS using measures of cortical morphology in whole brain T1 MR images.

We describe here a CAD system (software package) that can be used to identify the characteristics and patterns of MTS from brain magnetic resonance images. The image processing techniques involve texture analysis, statistical features, evaluation of the 3D region of interest (ROI), and threshold analysis using the T2 coronal images and FLAIR modalities of MRI. The software, allows the automatic evaluation of the degeneration of hippocampal structures, hippocampal volume and, signal intensity.

\section{METHODS}

With the automatic detection of the hippocampus in mind, an efficient algorithm has been developed for detection and identification of features of MTS $^{13}$. Figure 1 summarizes the methodology of this process.

\subsection{Data and Preprocessing}

The data used in this work comes from two sources: (1)Data from public sources, such as Kaggle ${ }^{26}$, and Medical Segmentation Decathlon ${ }^{27}$ and (2) anonymized data collected from a University Hospital in Loja Ecuador. This dataset consisted of images from 30 healthy patients adults (both males and females) as well as images from 70 patients exhibiting some neurological disorder (45 - mesial temporal sclerosis, 15 - other lesions such as ischemia or stroke, 10 brain tumors). These T1-weighted, T2-weighted, and FLAIR images were acquired with a RM Philips Medical Systems, Achieva model, with a field strength of 1.5 Teslas. Pre-processing consisted of eliminating artifacts in the images and conversion of the Dicom images to the nifty scheme. The nifty format allows for streamlining of the various training stage.

\subsection{Hippocampus Segmentation algorithm}

Image Analyzer software allows the user to choose multiple regions of interest (ROIs) in a set of images. That tool is used to determine the feature masks of the hippocampal volume and structures.

The segmentation algorithm consists of the extraction of various characteristics from the hippocampal masks. The masks have been previously classified according to the hemisphere to which they correspond, be it right or left. The masks correspond to the axial, sagittal and coronal slices of the MRI images. The three-dimensional grouping of twodimensional masks allows for recreation of the organ of interest, namely the hippocampus.

Through various post-processing algorithms such as texture smoothing, the volumetric body is reconstructed. The volume is therefore the sum of nonzero voxels within the Tensor space. In addition to this parameter, the signal intensity obtained with the volumetric surface is also added.

For the segmentation, ${ }^{28,29}$ well-known networks such as U-Net ${ }^{28}$, Unet3D, Vnet3D have been used in versions of PyTorch $^{29}$. The Image Analyzer software uses the U-Net network for each slice of the volumetric images, based on previous work ${ }^{30,31}$ (Figure 3). Image masks corresponding to the hippocampus that were previously segmented were taken from the datasets. 
After preprocessing the image is input to the 3D U-Net network to segment automatically the hippocampus. The size of input image is 128X128X64. The 3D U-Net parameters used were: batch normalization 64, 32 filters, 1 channel, and max pooling $2 \mathrm{X} 2$, up-convolution $2 \mathrm{X} 2$, activation function ReLU.

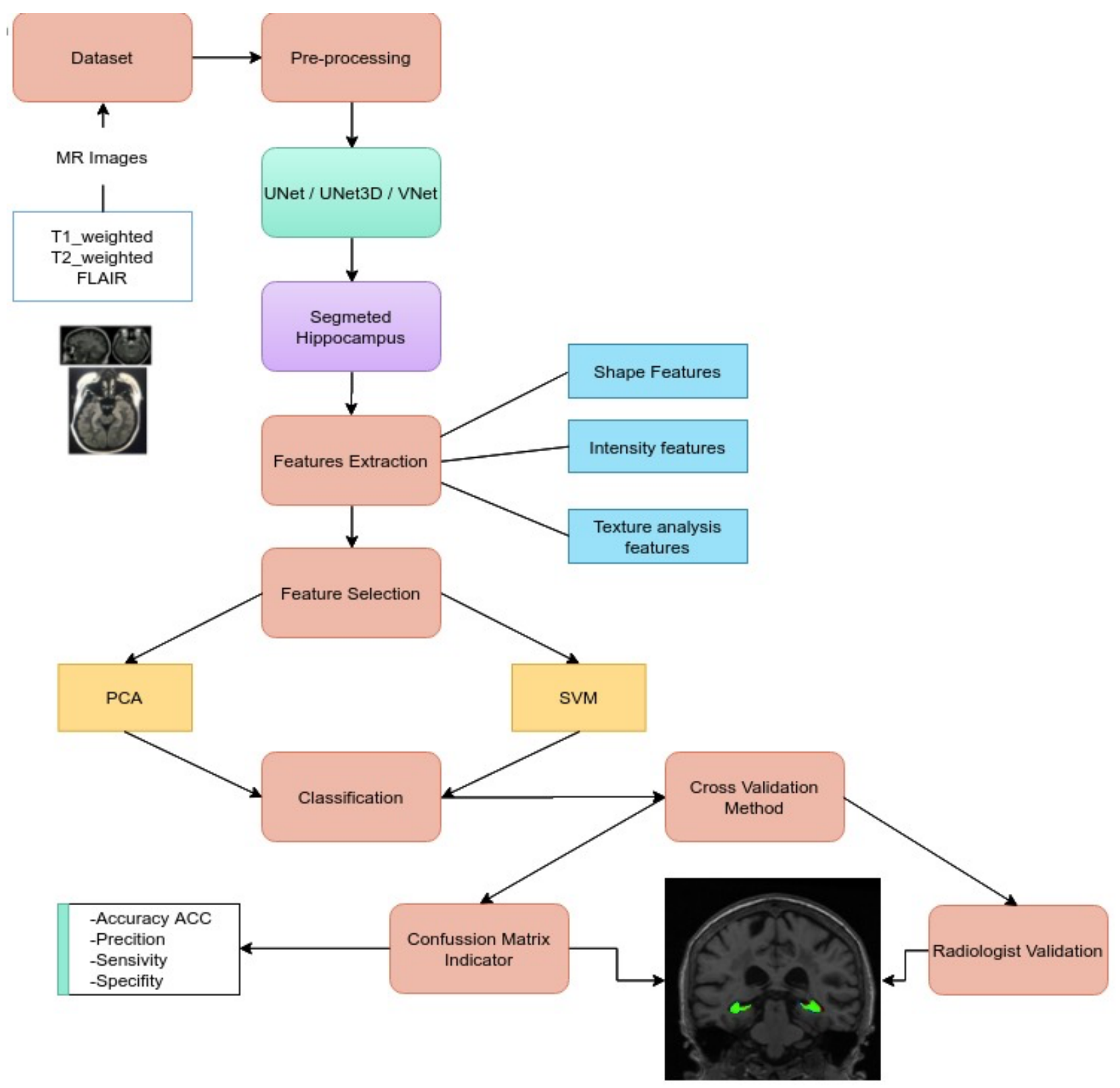

Figure 1. Methodology for segmentation and detection of hippocampus structure in the MR brain images. The algorithm consists of data collection, preprocessing, segmentation, features extraction and selection, classification and validation. 

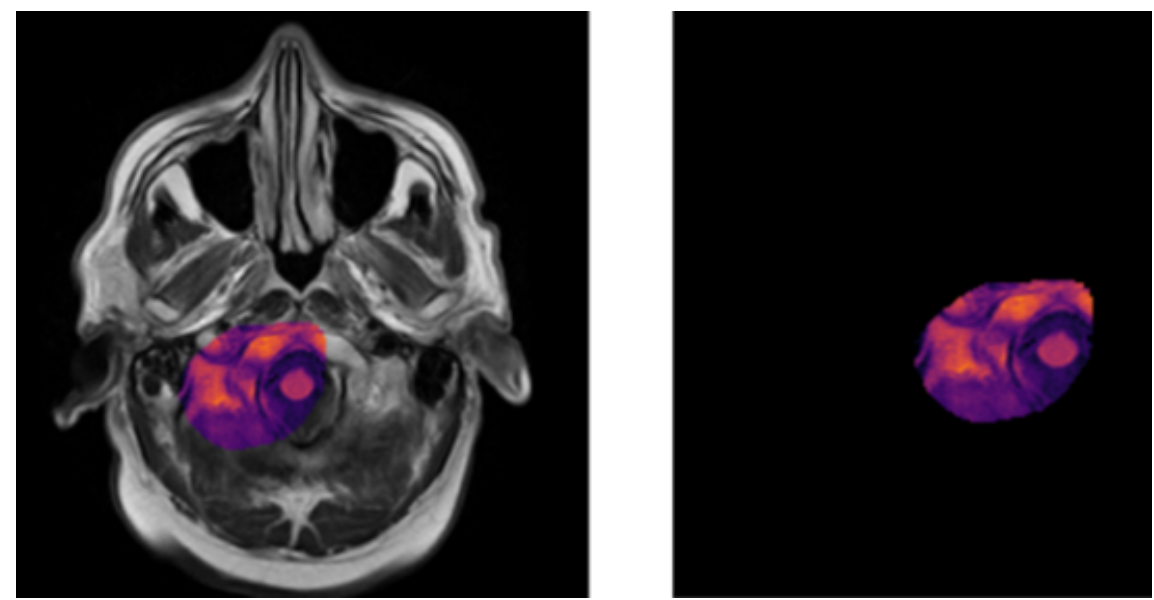

Figure 2. Image Analyzer, manual ROI selection to determine the mask features

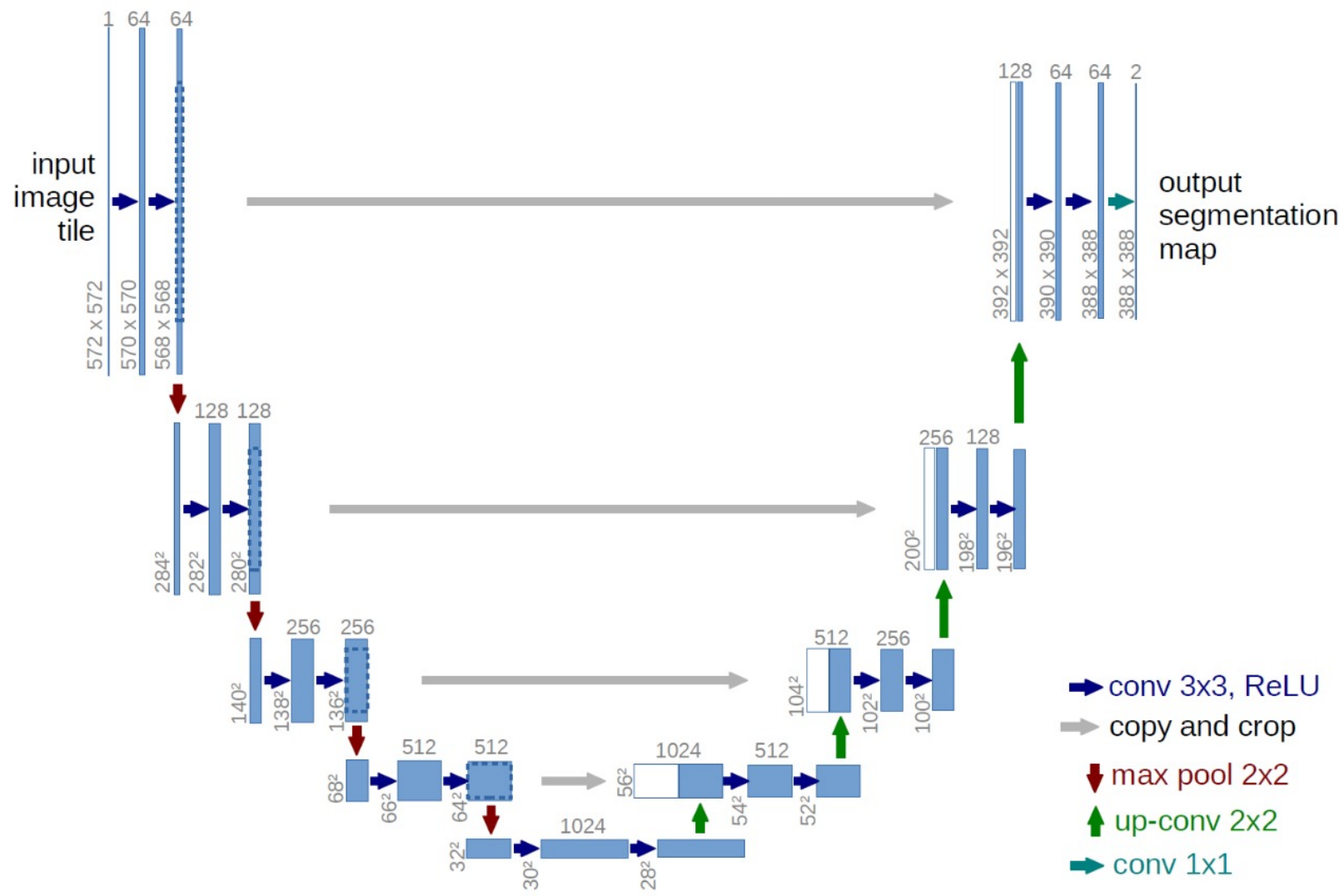

Figure 3. Classic scheme of the U-Net network. ${ }^{28}$ This was used in each slice of the volumetric brain image by the Image Analyzer software.

\section{RESULTS}

The image analyzer software package has been tested and the partial results are provided here. The partial results include the dataset collection, preprocessing and the analysis of the features extraction through the definitions of ROIs and the data for hippocampus volume and intensity signal. The Image Analyzer code is available at the GitHub site (https://github.com/Hikki12/image-analyzer). The user interface is shown in Figure 4. 


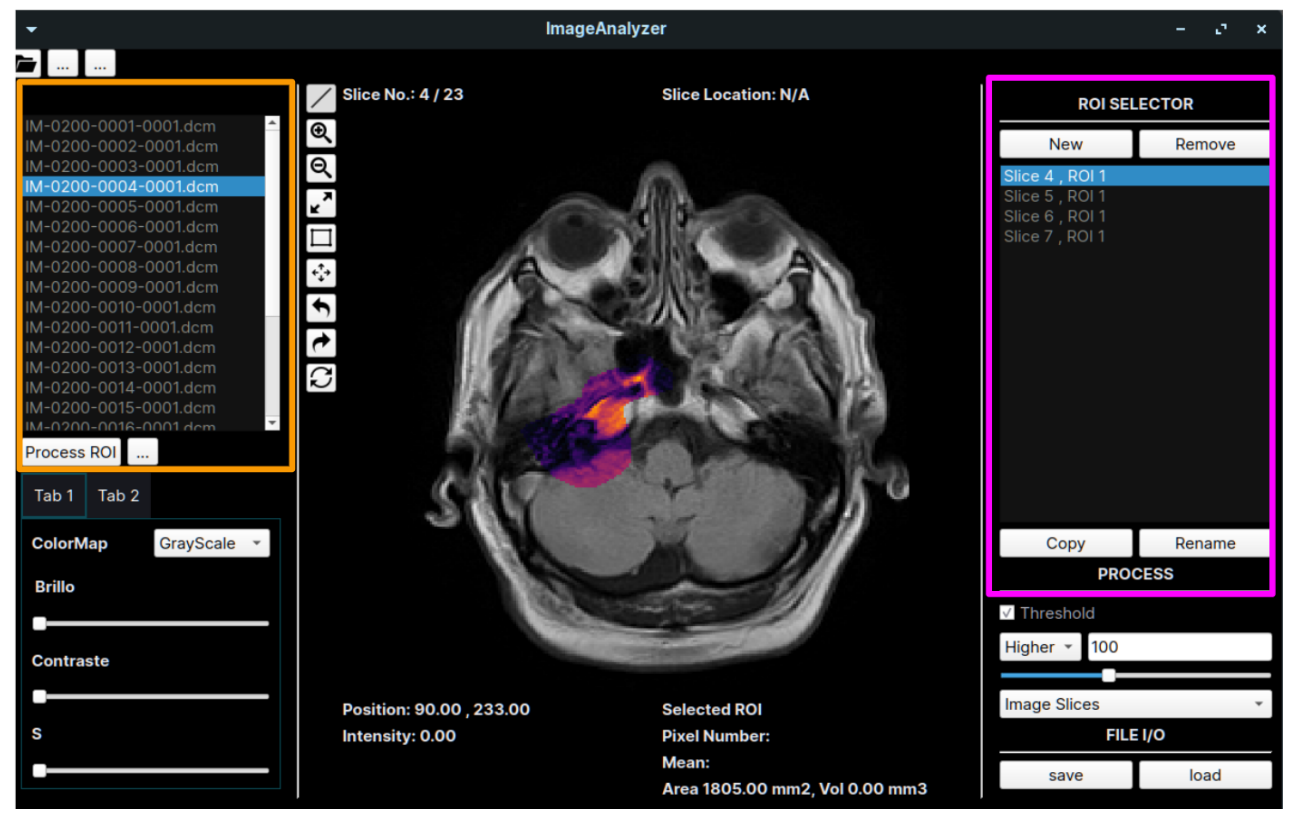

Figure 4. The user Interface of the Image Analyzer software package.

\subsection{ROI definition and Segmentation}

The Image Analyzer software package allows for manual segmentation using ROI and the automatic segmentation through 3D U-Net. The manual ROI segmentation also allows for determination of which are used to train the U-net network (Figure 5). The manual segmentation allows the ROI selection through the threshold analysis (Figure 5).
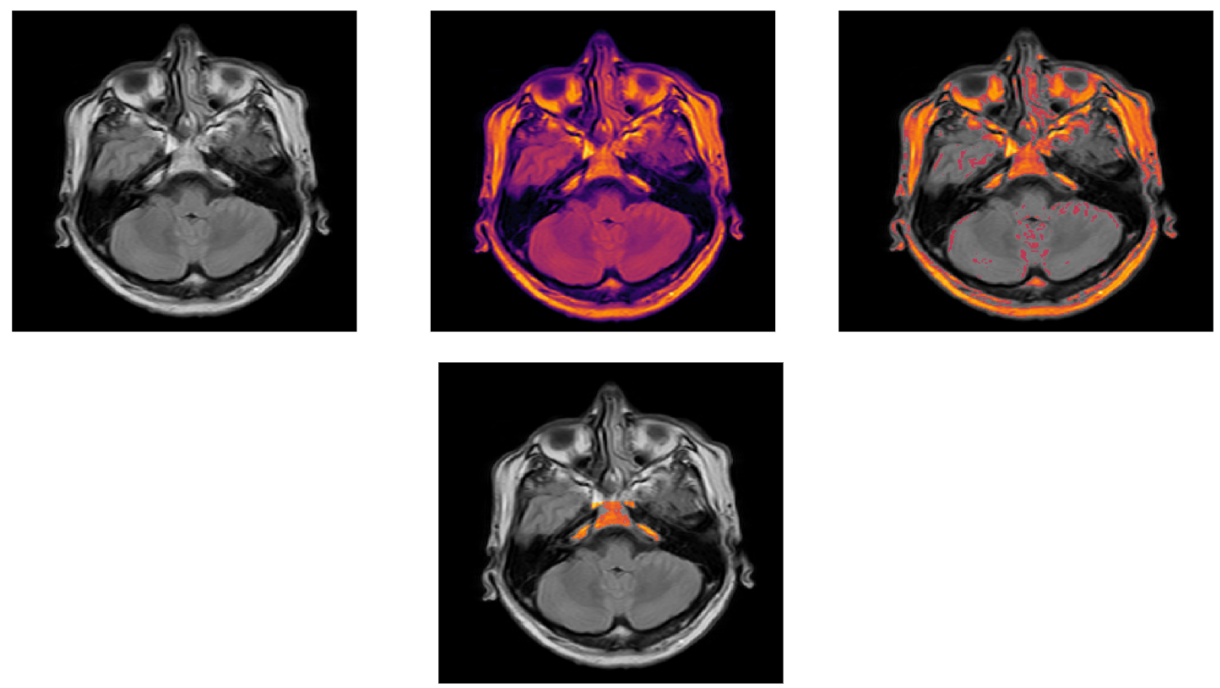

Figure 5. Manual ROI selection. Original image (top left), image with threshold 0-255 (top center), top right with threshold 20 to 250 , center bottom image with threshold 80 to 250 .

Once we get the segmented hippocampus, feature extraction and selection can be applied to train the SVM classifier in order to determine if the patient has MTS.

The selection and extraction of features is based in the statistical texture analysis ${ }^{32,33}$, which consist of the selection of the most relevant features through the intensity histogram of the segmented hippocampus. The texture definition is using the wavelet transform and features parameters like entropy, energy (signal intensity), shape-based (2D), shape-based (3D), and statistical parameters: Gray Level Cooccurrence Matrix (GLCM), Gray Level Run Length Matrix (GLRLM), 
Gray Level Size Zone Matrix (GLSZM), Neighbouring Gray Tone Difference Matrix (NGTDM) ${ }^{34}$. These parameters allow for characterization of the hippocampus volume and its relation to the MTS.
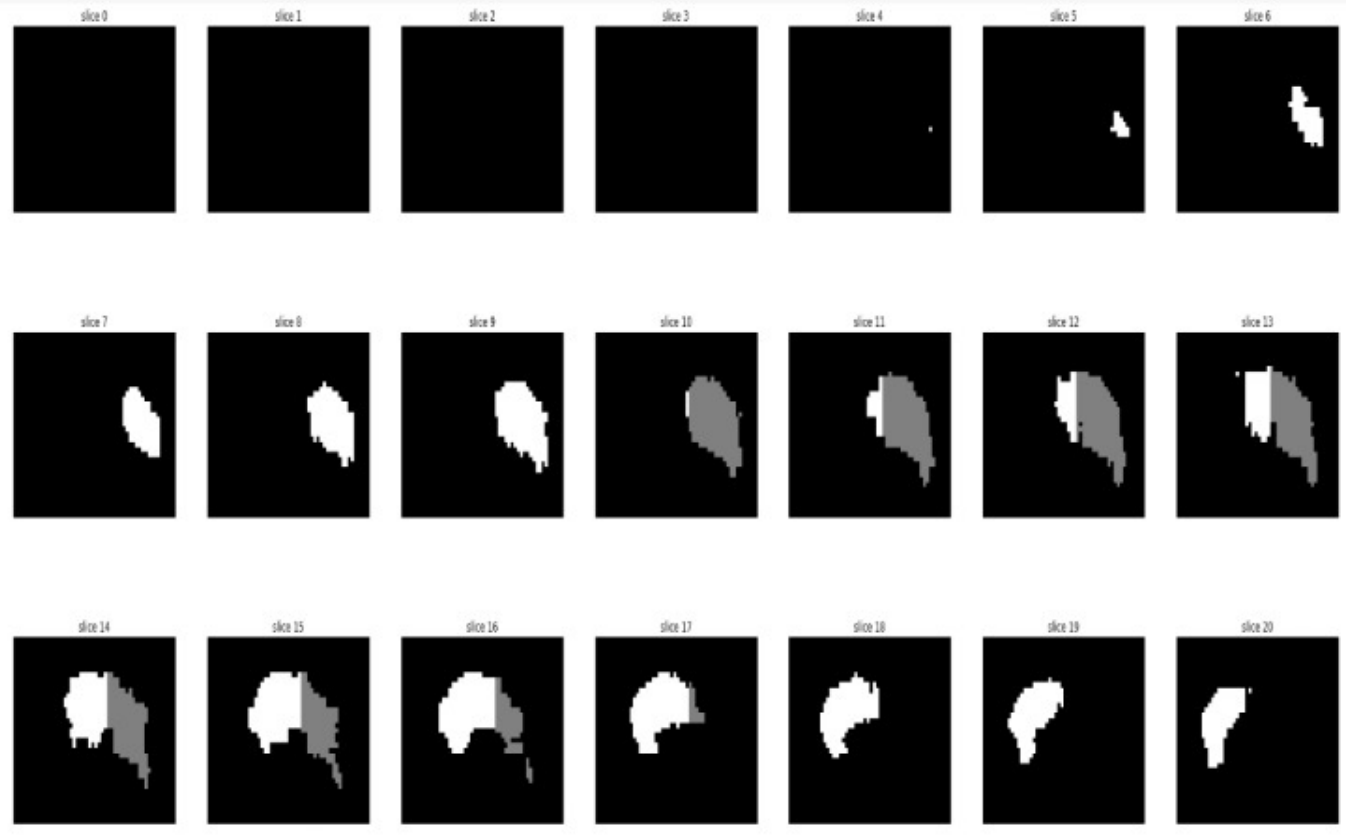

Figure 6. Hippocampal masks in various slices. These were obtained from datasets to train the U-Net 3D network.

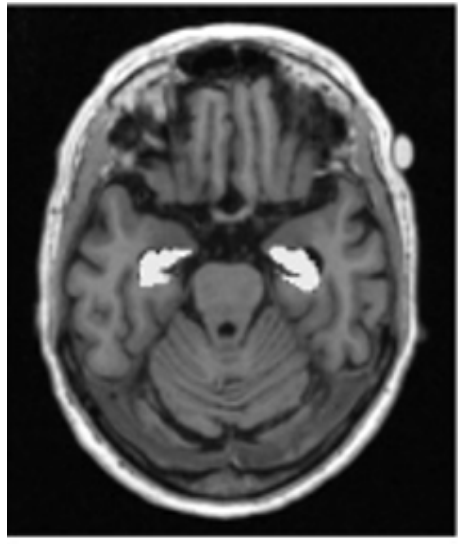

(a)

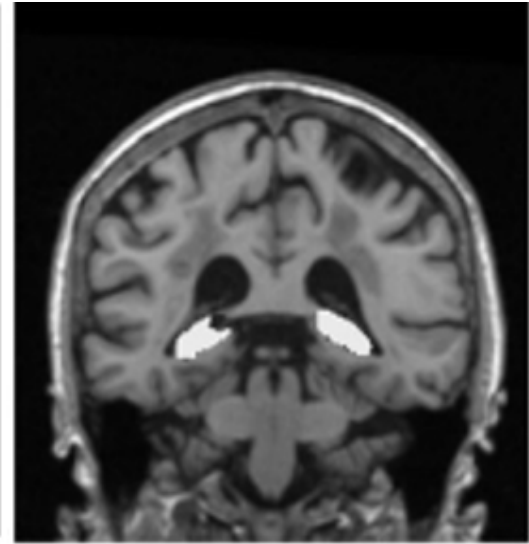

(b)

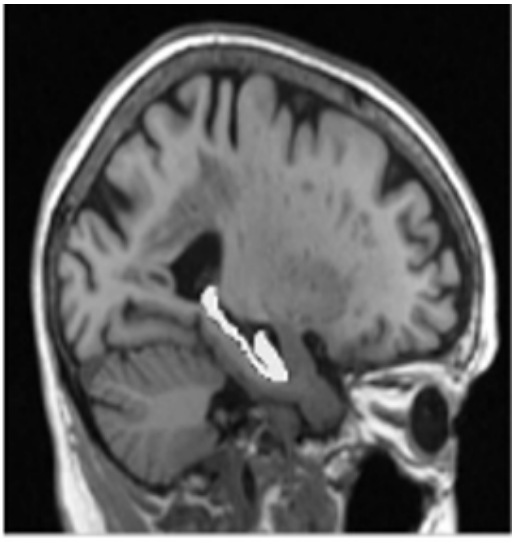

(c)

Figure 7. Automatic segmented hippocampus in different planes (a)axial (b)coronal, (c) sagittal.

\section{CONCLUSIONS}

The partial results show that the use of textures is a good tool for feature selection and segmentation of the hippocampus structure as well as for measurement of the volume employing the U-Net network. The 3D segmentation allows for significant segmentation which allows for determination of a good criterion to finding the MTS volume of the hippocampus and the variation of the signal and intensity in the anatomical structures.

It is expected that this tool will serve to train new neurology/radiology specialists and can be a source of secondary diagnostic opinion. However, it is necessary to validate the software system qualitatively and quantitatively in order to determine the effectiveness and efficiency in real-world clinical applications. This is the subject of future work. 


\section{REFERENCES}

[1] "Epilepsy.", <https://www.who.int/news-room/fact-sheets/detail/epilepsy> (28 June 2021).

[2] Malmgren, K. and Thom, M., "Hippocampal Sclerosis-Origins and imaging," Epilepsia 53(SUPPL. 4), 19-33 (2012).

[3] Thom, M., "Review: Hippocampal sclerosis in epilepsy: A neuropathology review," Neuropathology and Applied Neurobiology 40(5), 520-543 (2014).

[4] "Neuroimaging of epilepsy.", <https://www.ncbi.nlm.nih.gov/pmc/articles/PMC5256664/> (12 August 2020).

[5] Blümcke, I., Pauli, E., Clusmann, H., Schramm, J., Becker, A., Elger, C., Merschhemke, M., Meencke, H.-J., Lehmann, T., Von Deimling, A., Scheiwe, C., Zentner, J., Volk, B., Romstöck, J., Stefan, H. and Hildebrandt, M., "A new clinico-pathological classification system for mesial temporal sclerosis," Acta Neuropathologica 113(3), 235-244 (2007).

[6] Hogan, R. E., Wang, L., Bertrand, M. E., Willmore, L. J., Bucholz, R. D., Nassif, A. S. and Csernansky, J. G., "MRI-based high-dimensional hippocampal mapping in mesial temporal lobe epilepsy," Brain 127(8), 17311740 (2004).

[7] Azab, M., Carone, M., Ying, S. H. and Yousem, D. M., "Mesial Temporal Sclerosis: Accuracy of NeuroQuant versus Neuroradiologist," AJNR Am J Neuroradiol 36(8), 1400-1406 (2015).

[8] Gaillard, F., "Mesial temporal sclerosis | Radiology Reference Article | Radiopaedia.org," Radiopaedia, $<$ https://radiopaedia.org/articles/mesial-temporal-sclerosis $>$ (28 June 2021).

[9] Yamanakkanavar, N., Choi, J. Y. and Lee, B., "MRI Segmentation and Classification of Human Brain Using Deep Learning for Diagnosis of Alzheimer's Disease: A Survey," Sensors (Basel) 20(11), 3243 (2020) [doi:10.3390/s20113243].

[10] Nataraja, V., Farook, A. S., Elangovan, V., Ahmed, A. and Magudeeswaran, P. K., "Magnetic Resonance Imaging Evaluation of Hippocampus with T2 Relaxation Time," Int. J. Sci. Study 5(1), 116-120 (2017).

[11] Silva, G., Martins, C., Moreira da Silva, N., Vieira, D., Costa, D., Rego, R., Fonseca, J. and Silva Cunha, J. P., "Automated volumetry of hippocampus is useful to confirm unilateral mesial temporal sclerosis in patients with radiologically positive findings," Neuroradiology Journal 30(4), 318-323 (2017).

[12] Coan, A. C., Kubota, B., Bergo, F. P. G., Campos, B. M. and Cendes, F., "3T MRI quantification of hippocampal volume and signal in mesial temporal lobe epilepsy improves detection of hippocampal sclerosis," AJNR Am J Neuroradiol 35(1), 77-83 (2014).

[13] Castillo, D., Samaniego, R., Jiménez, Y., Sánchez, A., Carrión, J. and Álvarez, M. J. R., “Assessment of mesial temporal sclerosis through MRI processing," Emerging Topics in Artificial Intelligence 2020 11469, 114690W, International Society for Optics and Photonics (2020).

[14] Wang, H., Ahmed, S. N. and Mandal, M., "Computer-aided detection of mesial temporal sclerosis based on hippocampus and cerebrospinal fluid features in MR images," Biocybernetics and Biomedical Engineering 39(1), 122-132 (2019).

[15] Castillo, D., Lakshminarayanan, V. and Rodríguez-Álvarez, M. J., "MR Images, Brain Lesions, and Deep Learning," 4, Applied Sciences 11(4), 1675 (2021) [doi:10.3390/app11041675].

[16] Castillo, D. P., Samaniego, R. J., Jimenez, Y., Cuenca, L. A., Vivanco, O. A., Alvarez-Gomez, J. M. and Rodriguez-Alvarez, M. J., "Identifying Demyelinating and Ischemia brain diseases through magnetic resonance images processing," Proc. 2019 IEEE Nucl. Sci. Symp. Med. Imaging Conf., NSS/MIC, IEEE, 884-886 (2019).

[17] Castillo, D., Samaniego, R., Jiménez, Y., Cuenca, L., Vivanco, O. and Rodríguez-Álvarez, M. J., "Demyelinating and ischemic brain diseases: Detection algorithm through regular magnetic resonance images," Proc SPIE 10396, 103961C (2017).

[18] Singh, A., Sengupta, S. and Lakshminarayanan, V., "Glaucoma diagnosis using transfer learning methods," Applications of Machine Learning, M. E. Zelinski, T. M. Taha, J. Howe, A. A. Awwal, and K. M. Iftekharuddin, Eds., Proc. SPIE 11139, 111390U doi: 10.1117/12.2529429, 2019. (2019).

[19] Raghavendra, U., Acharya, U. R. and Adeli, H., "Artificial Intelligence Techniques for Automated Diagnosis of Neurological Disorders," Eur. Neurol. 82(1-3), 41-64 (2019).

[20] Mettenburg, J. M., Branstetter, B. F., Wiley, C. A., Lee, P. and Richardson, R. M., "Improved detection of subtle mesial temporal sclerosis: Validation of a commercially available software for automated segmentation of hippocampal volume," American Journal of Neuroradiology 40(3), 440-445 (2019). 
[21]Dahi, F., Parsons, M. S., Orlowski, H. L. P., Salter, A., Dahiya, S. and Sharma, A., "Image processing to improve detection of mesial temporal sclerosis in adults," American Journal of Neuroradiology 40(5), 798-801 (2019).

[22] Chupin, M., Hammers, A., Liu, R. S. N., Colliot, O., Burdett, J., Bardinet, E., Duncan, J. S., Garnero, L. and Lemieux, L., "Automatic segmentation of the hippocampus and the amygdala driven by hybrid constraints: Method and validation," Neuroimage 46(3), 749-761 (2009).

[23] Jafari-Khouzani, K., Elisevich, K., Patel, S., Smith, B. and Soltanian-Zadeh, H., "FLAIR signal and texture analysis for lateralizing mesial temporal lobe epilepsy," NeuroImage 49(2), 1559-1571 (2010).

[24] Cantor-Rivera, D., Khan, A. R., Goubran, M., Mirsattari, S. M. and Peters, T. M., "Detection of temporal lobe epilepsy using support vector machines in multi-parametric quantitative MR imaging," Computerized Medical Imaging and Graphics 41, 14-28 (2015).

[25] Rudie, J. D., Colby, J. B. and Salamon, N., "Machine learning classification of mesial temporal sclerosis in epilepsy patients," Epilepsy Research 117, 63-69 (2015).

[26] "Find Open Datasets and Machine Learning Projects | Kaggle.”, <https://www.kaggle.com/datasets> (29 June 2021).

[27] "Medical Segmentation Decathlon.", <http://medicaldecathlon.com/> (29 June 2021).

[28] Ronneberger, O., Fischer, P. and Brox, T., "U-Net: Convolutional Networks for Biomedical Image Segmentation," Medical Image Computing and Computer-Assisted Intervention - MICCAI 2015, N. Navab, J. Hornegger, W. M. Wells, and A. F. Frangi, Eds., 234-241, Springer International Publishing, Cham (2015).

[29] "The difference between UNet, 3D-UNet, VNet - Programmer Sought.", $<$ https://www.programmersought.com/article/68715704431/> (30 June 2021).

[30] Ataloglou, D., Dimou, A., Zarpalas, D. and Daras, P., "Fast and Precise Hippocampus Segmentation Through Deep Convolutional Neural Network Ensembles and Transfer Learning," Neuroinformatics 17(4), 563-582 (2019).

[31] Carmo, D., Silva, B., Yasuda, C., Rittner, L. and Lotufo, R., "Hippocampus segmentation on epilepsy and Alzheimer's disease studies with multiple convolutional neural networks," Heliyon 7(2), e06226 (2021).

[32] López-Gómez, C., Ortiz-Ramón, R., Mollá-Olmos, E. and Moratal, D., "ALTEA: A Software Tool for the Evaluation of New Biomarkers for Alzheimer's Disease by Means of Textures Analysis on Magnetic Resonance Images,” Diagnostics (Basel) 8(3), 47 (2018) [doi:10.3390/diagnostics8030047].

[33] Vallières, M., Freeman, C. R., Skamene, S. R. and Naqa, I. E., "A radiomics model from joint FDG-PET and MRI texture features for the prediction of lung metastases in soft-tissue sarcomas of the extremities," Phys. Med. Biol. 60(14), 5471-5496 (2015).

[34] Amadasun, M. and King, R., "Textural features corresponding to textural properties," IEEE Trans. Syst., Man, Cybern. 19(5), 1264-1274 (1989). 